\title{
LncRNA NORAD functions as a competing endogenous RNA to regulate KMT2D expression by targeting miR-204-5p in gastric cancer
}

Yan Zhou ( $\nabla$ yan_zhou09@163.com)

Nanfang Hospital, Southern Medical University

\section{Zhenwei Deng}

Dongguan People's Hospital, Southern Medical University

Ting Pan

Dongguan People's Hospital, Southern Medical University

\section{Guohao Chen}

Dongguan People's Hospital, Southern Medical University

\section{Yongchang Cai}

Dongguan People's Hospital, Southern Medical University

\section{Yuxin Tang}

Dongguan People's Hospital, Southern Medical University

\section{Yijun Wang}

Dongguan People's Hospital, Southern Medical University

\section{Yucheng Wang}

Dongguan People's Hospital, Southern Medical University

\section{Ruiping Li}

Dongguan People's Hospital, Southern Medical University Libo Li

Dongguan People's Hospital, Southern Medical University

\section{Research Article}

Keywords: NORAD, gastric cancer, miR-204-5p, competing endogenous RNA

Posted Date: February 11th, 2022

DOI: https://doi.org/10.21203/rs.3.rs-1342705/v1

License: (1) (1) This work is licensed under a Creative Commons Attribution 4.0 International License.

Read Full License 


\section{Abstract}

Background: Long noncoding RNAs (IncRNAs) are a class of noncoding RNAs implicated in the development of many malignancies, including gastric cancer (GC). Herein, we investigated the functions and molecular mechanisms of non-coding RNA activated by DNA damage (NORAD) in GC.

Methods: NORAD expression at the mRNA levels was determined by qRT-PCR assay. Cell proliferation, migration and invasion was detected by CCK-8 assay, in vivo tumor formation assay and Transwell assay. NORAD location was detected by nucleocytoplasmic fractionation assay. The interaction between NORAD and miR-204-5p/KMT2D axis was verified by dual-luciferase reporter gene assay and RIP assay. Results: NORAD was upregulated in GC tissues and cell lines. NORAD silencing repressed cell proliferation and G2/M cell cycle transition in GC. NORAD could act as a ceRNA of miR-204-5p and regulate KMT2D expression.

Conclusions: Our study demonstrated that NORAD played as an oncogene in GC, which might provide a new therapeutic target for the disease.

\section{Introduction}

Gastric cancer (GC) is s one of the most common malignant tumors worldwide, constituting the sixth leading cause of cancer related- mortality $(1,2)$. As most GC patients are at an advanced stage when diagnosed and the 5-year survival rates remain

low, particularly for those with local and distant metastasis $(3,4)$. Improved GC early diagnosis and survival outcomes is thus urgently needed.

Protein coding genes regulate many cell processes including differentiation, metabolism and tumorigenesis and have been well-studied in recent years $(5,6)$. However, with the development of human genome sequencing, non-coding RNA (ncRNA) has gradually attracted increasing attention $(7,8)$. Emerging evidence suggests that IncRNAs are key players a multitude of cellular processes, especially resulting in aberrant expression of gene products link to the advance of diverse human cancers $(9,10)$. However, the role of IncRNAs and its molecular mechanism involved in GC is still largely unclear.

Non-coding RNA activated by DNA damage (NORAD), a newly discovered IncRNA that maps to 20q11.23 in human, has been reported as an oncogenic factor in numbers of human cancers(11-16). For instance, a recent study revealed that NORAD was upregulated in GC tissues and promoted GC cell growth in vitro(17). Another report indicated that silencing of NORAD suppressed cell proliferation and invasion via positive modulation of RhoA/ROCK1 pathway in GC(18). Micro-RNAs (miRNAs) are a type of endogenous short-chain non-coding RNA molecules that involved in the development and progression of human cancers $(19,20)$. Acting as competitive endogenous RNAs (ceRNAs) to sponge miRNAs is one of the most well-established biological roles of IncRNAs $(21,22)$. Interestingly, NORAD also functioned as a ceRNA in many cancers(23-26). MiR-204-5p is a miRNA implicated in the development and progression of human 
cancers, including GC(27-30). Given that there are limited studies that reveals the relationship between NORAD and miR-204-5p in GC. In the present study, we aimed to comprehensively elucidate the functional roles of NORAD/miR-204-5p axis and their molecular mechanisms in GC, as well as their potential as therapeutic target for the disease.

\section{Materials And Methods Clinical samples}

Our sample cohort contained a total of 60 pairs of matched normal and GC tissues, which were obtained from Affiliated Dongguan People's Hospital, Southern Medical University (Dongguan People's Hospital). All the tissue samples after liquid nitrogen refrigeration were saved in the- $80^{\circ} \mathrm{C}$ cryogenic refrigerator. All subjects have written informed consent. This study was approved by the Ethics Committee of Dongguan People's Hospital, Southern Medical University (KYKT-2020-026) and that this was conducted in accordance with the Declaration of Helsinki.

\section{Quantitative qRT-PCR (qRT-PCR) assay}

Cellular RNAs were extracted using Trizol Reagent (Invitrogen, CA, USA) according to the manufacturer's instructions. PrimeScript RT Reagent Kit (TaKaRa Biotechnology, Dalian, China) was used for total RNA reverse transcription. The expression of NORAD and KMT2D was detected by a LightCycler 480 Instrument (Roche, Basel, Switzerland), using SYBR Green PCR Kit (TaKaRa Biotechnology) and GAPDH was used as the internal control. Relative expression of miR-204-5p was measured using TaqMan MicroRNA Assays (Applied Biosystems) and U6 was treated as an internal control. All the primer sequences were listed in Table 1.

\section{Table 1}

The sequences of the primers. 


\begin{tabular}{ll} 
Gene name & Primer sequences \\
\hline NORAD forward primer & 5- $C T G G A T G \forall G G C A G A G \forall G G \forall G G$ - 3 \\
\hline NORAD reverse primer & 5- T GTC $A \mathbb{C} A C A T A C A C A G C A C T G$ - 3 \\
\hline GAPDH forward primer & 5-GTCAACGGATTTGGTCTGTATT-3 \\
\hline GAPDH reverse primer & 5-AGTCTTCTGGGTGGCAGTGAT-3 \\
\hline miR-204-5p forward primer & 5-ACTATGGCTTCCCTTTGTCATCC-3 \\
\hline miR-204-5p reverse primer & 5-AGTGCAGGGTCCGAGGTATT-3 \\
\hline U6 forward primer & 5-CTCGCTTCGGCAGCACA-3 \\
\hline U6 reverse primer & 5-AACGCTTCACGAATTTGCGT-3 \\
\hline KMT2D forward primer & 5-TGACAAGTGTGAATCCCGTGAAG-3 \\
\hline KMT2D reverse primer & 5-CATTTCATCCGTTGTTACGAAG-3
\end{tabular}

\section{Cell culture}

Cell lines including MGC-803, SGC-7901, BGC-823, MKN-45, AGS and GES-1 were purchased from Chinese Academy of Sciences (Shanghai, China). MGC-803, BGC-823 and MKN-45 cells were cultured with RPMI 1640. SGC-7901, AGS and GES-1 cells were cultured with DMEM supplemented with $10 \%$ fetal bovine serum (FBS; Gibco, Rockville, MD, USA), $100 \mathrm{U} / \mathrm{ml}$ penicillin, and $100 \mathrm{mg} / \mathrm{ml}$ streptomycin (Invitrogen,) at $37^{\circ} \mathrm{C}$ in $5 \% \mathrm{CO}_{2}$.

\section{Cell transfection}

Short hairpin RNA (shRNA) and a NC shRNA specific for NORAD were obtained from Sangon Biotech (Guangzhou, China). They infected GC cells with a mixture of the lentiviruses (multiplicity of infection [MOI], 100) and $5 \mathrm{mg} / \mathrm{mL}$ polybrene. miR-204-5p angomir/NC and miR-204-5p antangomir/NC were synthesized by GenePharma Co., Ltd. (Shanghai, China). They were transfected into GC cells using Lipofectamine 3000 (Invitrogen, Carlsbad, CA, USA) according to the manufacturer's instructions.

\section{Cell Counting Kit (CCK-8) assay}

Cells were placed in 96-well plates at cell density of $2 \times 10^{3}$ cells/well. At the appointed time points $(24,48$, $72 \mathrm{~h}$ ), $10 \mu \mathrm{L}$ of Cell Counting Kit-8 solution (CCK-8; Dojindo, Kumamoto, Japan) was added in each well. Absorbance at $450 \mathrm{~nm}$ was taken for each sample using a micro-plate reader (Bio-Rad, Hercules, CA, USA). 


\section{Cell cycle assay}

Cells were fixed using 70\% cold anhydrous ethanol. Then, cells were treated with PI (KeyGen Biotech, Nanjing, China) with RNase A. Cell cycle distribution was analyzed using a flow cytometer (BD Biosciences, San Diego, CA, USA).

\section{In vivo tumor formation assay}

Lentiviral-mediated stably NORAD-knockdown cells and negative control cells were subcutaneously injected into the flanks of BALB/c nude mice (SPF grade, 3-4 weeks old, male). The tumor volume was measured every 4 days. The volume of xenografted tumor was monitored every 3 days using the following formula: Volume $=($ length $\times$ width $\times$ width $) / 2$. The animal study was conducted with the approval of the Institutional Animal Care and Use Committee of the Affiliated Dongguan People's Hospital, Southern Medical University (Dongguan People's Hospital) (KYKT-2020-026).

\section{Transwell assay}

Cells were added to the top chamber without or with Matrigel coating while lower chamber was added with culture medium containing $10 \%$ FBS only. Following $24 \mathrm{~h}$ cell culture, cells migrating or invading the lower chamber were fixed using $4 \%$ paraformaldehyde, stained with $0.1 \%$ crystal violet solution and subsequently visualized using inverted light microscope.

\section{Nucleocytoplasmic fractionation assay}

The cytoplasmic and nuclear components were separates using PARIS Kit (Life Technologies, MA, USA). The nuclear and cytoplasmic fractions was detected by qRT-PCR assay to determine NORAD distribution using GAPDH and U6 as the cytoplasmic reference and nuclear reference, respectively.

\section{Bioinformatics analysis and dual-luciferase reporter gene assay}

The binding sites of miR-204-5p with NORAD-3UTR and KMT2D-3UTR were obtained from Starbase. Putative wild-type (WT) and mutant (MUT) miR-204-5p -binding sites in the 3囚-UTR of NORAD or KMT2D mRNA, termed NORAD-WT or NORAD-MUT and KMT2D-WT or KMT2D-MUT, were sub-cloned into pGL3 Basic vector (Promega, Madison, WI, USA). miR-204-5p angomir or angomir NC were co-transfected with NORAD-WT or NORAD-MUT and KMT2D-WT or KMT2D-MUT reporter constructs using Lipofectamine $\mathrm{e}^{\mathrm{TM}} 3000$ agent. Luciferase activity was determined using the Multimode Detector reporter assay system (Beckman Coulter, WI, USA). 


\section{RNA immunoprecipitation (RIP) assay}

RIP was performed using a Magna RIP RNA-Binding Protein Immunoprecipitation kit (Millipore, Billerica, MA, USA) according to the manufacturer's instructions. An anti-Argonaute-2 (Ago-2) or anti-IgG antibodies was also used. Enrichment of NORAD and miR-204-5p was determined by qRT-PCR assay.

\section{Western blot}

Cells lysates extracted using radioimmunoprecipitation assay (RIPA) (Beyotime, Shanghai, China) were fractionated by electrophoresis and transferred on a polyvinylidene fluoride (PVDF) membrane (Millipore). The membrane was blocked in 5\% nonfat skim milk and incubated with primary antibodies and secondary antibodies. Bands on the membrane were visualized by electrochemiluminescence (ECL; Pierce, Rockford, IL, USA). The primary antibodies used were as follows: anti-PTEN (1:1000), anti-PTEN (1:1000), anti-PI3K (1:1000), anti-AKT (1:1000), anti-phosphorylated-AKT (pAKT; 1:1000), (Cell Signaling Technology, Danvers, CO, USA).

\section{Statistical analysis}

All statistical analysis were performed using SPSS 21.0 (SPSS, Chicago, IL, USA) or GraphPad Prism (GraphPad Prism, Inc., La Jolla, CA, USA). Each experiment was performed in three independent replicates and the data are presented as the mean \pm standard deviation (SD). Student's t-test or one-way ANOVA was used to compare the means of two or three groups. $P<0.05$ was considered statistically significant.

\section{Results}

\section{NORAD was highly expressed in GC tissues and cell lines}

A cohort containing 60 pairs of fresh frozen GC tissues and paired adjacent normal tissues was involved in our study. qRT-PCR assay was performed to determine different expression levels of NORAD. As shown in Fig. 1A, NORAD was obviously upregulated in the GC tissues compared with the matched adjacent normal tissues $(P<0.001)$. We also examined the expression of NORAD in different cell lines. The results showed that the expression of NORAD was significantly higher in several GC cell lines (MKN-45, SGC7901, AGS, BGC-823, MGC-803) than in the gastric epithelium cell line (GES-1) (Fig. 1B). Among these cell lines, MKN-45 and SGC-7901 cells exhibited the highest level and were employed in the following experiments. Collectively, these results indicate that NORAD is highly expressed in GC.

\section{NORAD silencing hampered GC cell growth in vitro and in vivo}


To evaluate the biological roles of NORAD in the behaviors of GC cells, two shRNAs specifically targeting NORAD (shNORAD-1 and shNORAD-2) were designed and constructed. They were transfected into MKN45 and SGC-7901 cells, respectively. qRT-PCR assay showed that shNORAD-1 and shNORAD-2 could significantly reduce the expression levels of NORAD in MKN-45 and SGC-7901 cells (Fig. 2A, B), which indicated a satisfactory interfering efficiency for further loss-function experiments.

Sustained proliferative signaling is one of the hallmarks of cancer. The role of NORAD silencing on cell growth in GC were firstly investigated. The results of CCK-8 demonstrated that cell proliferation was obviously suppressed in shNORAD group compared with shControl group (Fig. 2C, D). We then evaluated the effect of NORAD on GC growth in mouse models. As shown in Fig. 2E, F, the size and weight of tumors in shNORAD silencing group were significantly lower than those in shControl group at 18 days after inoculation.

\section{NORAD silencing leads to cell cycle arrest but did not affect cell migration and invasion in GC}

Uncontrolled cell division contributes to the malignant characteristic of cancer cells. To determine whether aberrant NORAD expression disturbs cell cycle, we analyzed the mitotic cycle of GC cells with NORAD silencing. Flow cytometric analysis showed that the proportion of MKN-45 and SGC-7901 cells increased remarkably at $\mathrm{G} 2 / \mathrm{M}$ phase of mitosis upon NORAD knockdown $(P<0.05$, Fig. 3A, B).

Activating invasion and metastasis is another malignant characteristic of cancer cells. The ability of cell invasion and migration were assessed by Transwell assay. As shown in Fig. S1A, transfection with shNORAD did not affect GC cell invasion in contrast with transfection with shControl. The changes of cell migratory ability had the same trend with cell invasion (Fig. S1B). These results implicated that NORAD silencing arrested cell cycle, but not changed cell invasion or migration in GC.

\section{NORAD could bind to miR-204-5p}

Cytoplasmic IncRNAs are more often involved in post-transcriptional regulation, such as playing the role of miRNA sponges. In the present study, we determined the cytoplasmic localization of NORAD in GC cells (Fig. 4A, B). As NORAD was mainly colocalized in cytoplasm, we further analyzed whether it could post-transcriptionally regulate gene expression by acting as a ceRNA. Firstly, miR-204-5p was predicted as a target of NORAD and their putative binding sites were obtained (Fig. 4C). Moreover, to determine the interaction between NORAD and miR-204-5p, a dual-luciferase reporter gene assay was performed in MKN-45 and SGC-7901 cells. The results showed that overexpression of miR-204-5p significantly reduced the luciferase activity of wild-type NORAD (NORAD WT) vector but did not change the luciferase activity of wild-type NORAD (NORAD WT) vector indicating the interaction between NORAD and miR-204-5p. RIP assay further confirmed that NORAD can bind to miR-204-5p, as higher levels of NORAD and miR-204-5p 
in the Ago2 antibody group than in the IgG control group (Fig. 4F, G). Subsequently, qRT-qPCR assay showed that NORAD silencing increased miR-204-5p expression levels (Fig. 4H, I). miR-204-5p expression was found to be lower in GC tissues (Fig. 4J). Furthermore, a negative correlation between NORAD and miR-204-5p expression in GC tissues was determined by Pearson correlation analysis (Fig. 4K). In all, our data suggested that NORAD could directly bind to miR-204-5p.

\section{KMT2D is a target gene of miR-204-5p in GC}

Based on bioinformatic prediction, miR-204-5p could target KMT2D (Fig. 5A). Their interaction was then confirmed by a dual-luciferase reporter gene assay. The results showed that the luciferase activity of MKN-45 and SGC-7901 cells with KMT2D WT vector decreased upon overexpression of miR-204-5p, whereas no significant change was observed in MKN-45 and SGC-7901 cells with KMT2D MUT vector (Fig.5B, C). Moreover, overexpression of miR-204-5p dramatically reduced KMT2D mRNA and protein expression in MKN-45 and SGC-7901 cells (Fig. 5D, E). We also confirmed a statistical negative correlation between KMT2D mRNA and protein expression in GC tissues $(r=-0.3674, P<0.001$; Fig. $5 \mathrm{~F})$. Further qRT-PCR assay and Western blot uncovered that the mRNA and protein levels of KMT2D were suppressed in MKN-45 and SGC-7901 cells with NORAD knockdown (Fig. 5G, H). Finally, Pearson correlation analysis validated a positive correlation between NORAD and KMT2D mRNA expression in the same sample cohort (Fig. 5I). Taken together, NORAD might function as a ceRNA against miR-204-5p to regulate KMT2D.

\section{The inhibitory effects of NORAD silencing on GC cell growth were mediated by miR-204-5p/KMT2D axis}

Based on the above assays, we speculated that the NORAD/miR-204-5p/KMT2D axis played an important role in GC growth, therefore rescue assays were then performed. As shown in Fig. 6A-D, miR204-5p antangomir reversed the anti-proliferation effects on MKN-45 and SGC-7901 cells induced by NORAD silencing. In addition, NORAD silencing resulted in an increase of G2/M phase arrest while miR204-5p antangomir rescued the increase. However, such rescuing effects could be partially abolished by the co-transfection of siKMT2D. Based on the above results, we highly proved that NORAD/miR-204$5 \mathrm{p} / \mathrm{KMT} 2 \mathrm{D}$ axis exerts potential regulatory effects on GC.

\section{NORAD/miR-204-5p/KMT2D axis regulated PTEN/PI3K/AKT signaling pathway in GC}

Increasing information has suggested that PTEN/PI3K/AKT signaling pathway is a biological participator in tumor progression. In the present study, we investigated whether NORAD led to the aberrant activation of PTEN/PI3K/AKT signaling pathway in GC cells. We measured the protein levels of PTEN/PI3K/AKT 
pathway factors and found that NORAD silencing significantly increased the expression of PTEN, but reduced the expression of PI3K and phosphorylated AKT (pAKT), indicating the potential regulatory effect of NORAD on PTEN/PI3K/AKT signaling pathway. Such regulatory effect was also mediated by miR-2045p/KMT2D axis (Fig. 7).

\section{Discussion}

GC results from a multistep process through the accumulation of numerous genetic and epigenetic alterations in oncogenes and tumor suppressor genes $(31,32)$. LncRNAs were once considered to be simply cloning artifacts or transcriptional noise.(33); however, in recent years, multiple studies have highlighted the potential roles of IncRNAs in GC pathogenesis (34). The current study delineated that NORAD expression was elevated in GC tissues and cell lines, and silencing of NORAD suppressed the GC cells growth via induction of cell cycle arrestment, suggesting the potential role of this IncRNA in GC progression.

Our study found that NORAD post-transcriptionally regulated the progression of GC through the ceRNA mechanism. The ceRNA network hypothesis state that IncRNA can absorb miRNAs by binding to them and subsequently exhibiting a miRNA sponge function, finally constructing a framework to systematically functionalize miRNA-response elements (MRE)-harboring noncoding RNAs and integrate them with the protein-coding RNA dimension $(21,22)$. As reported previously, long intergenic nonprotein coding RNA 00184 (LINC00184) encourages proliferation and invasion of GC cells by targeting miR-145/ANGPT2 pathway(35). Chen et al. reported that IncRNA HCP5 inhibits GC progression via functioning as a ceRNA for miR-106b-5p to affect p21 expression(36). In line with these reports, our study found that NORAD interacted with miR-204-5p, which has been also reported in Parkinson's disease(37). The present data showed that KMT2D was validated as a downstream target of miR-204-5p. In our previous study, we found that KMT2D was overexpressed in GC tissues(38). Moreover, we showed that depletion of KMT2D suppressed cell proliferation in vitro and in vivo. In present study, we found that KMT2D had a negative correlation with miR-204-5p but a positive correlation with NORAD in GC tissues. Based on the luciferase reporter assay, we eventually verified a direct interaction between KMT2D and miR-204-5p in GC cells. Tus, we hypothesized that NORAD sponged miR-204-5p to regulate KMT2D in GC cells.

Phosphoinositide 3-kinases (PI3Ks), consisted in PI3K/AKT signaling pathway, a family of signaling enzymes which regulate diverse cellular processes including apoptosis, metabolism, cell proliferation and cell growth (39). The tumor suppressor PTEN, decreased or absent in many tumors, can block the biological processes to inhibit the development of tumors through inactivating PI3K/AKT signaling pathway(40). It has also been fully-studied that PTEN/PI3K/AKT signaling pathway is able to confer tumorigenic properties GC. In our previous study, KMT2D depletion triggered the changes in the PTEN/PI3K/AKT pathway(38). Another study reported by Lv et al. found that KMT2D sustained carcinogenesis by activating PI3K/AKT pathway(41). In the present study, NORAD, which mediated the expression of KMT2D by targeting miR-204-5p, was verified as a positive regulator of cell proliferation and cell cycle via PTEN/PI3K/AKT pathway in GC cells. Most importantly, rescue experiments further 
confirmed that NORAD regulated the malignant features by targeting miR-204-5p/KMT2D axis in GC cells. These data unveiled a novel role for NORAD as a therapeutic target for GC.

\section{Conclusions}

Our study identified NORAD as a promising oncogene in GC. NORAD contributed to GC growth through modulation of KMT2D expression via acting as a ceRNA for miR-204-5p. These findings enhance our knowledge of the role of NORAD and reveal new therapeutic strategies for GC.

\section{Declarations}

\section{Acknowledgements}

Not applicable.

\section{Authors' contributions}

$R L, L L$ and $Y Z$ designed and supervised the study. ZD and TP performed the experiments and acquired result data. GC, YC and YT helped to perform the partial experiments. YW helped to review the statistical analysis. ZD drafted the manuscript. ZD and TP critically revised the manuscript. All authors read and approved the final version of the manuscript.

\section{Funding}

Not applicable.

\section{Availability of data and materials}

The datasets used and/or analyzed during the current study are available from the corresponding author on reasonable request.

\section{Ethics approval and consent to participate}

The study was approved by the Ethics Committee of Dongguan People's Hospital, Southern Medical University. Signed written informed consents were obtained from the patients.

\section{Consent for publication}

Not applicable. 


\section{Competing interests}

The authors declare that they have no competing interests

\section{References}

1. Siegel RL, Miller KD, Fuchs HE, Jemal A. Cancer Statistics, 2021. CA Cancer J Clin. 2021;71(1):7-33.

2. Bray F, Ferlay J, Soerjomataram I, Siegel RL, Torre LA, Jemal A. Global cancer statistics 2018 : GLOBOCAN estimates of incidence and mortality worldwide for 36 cancers in 185 countries. CA Cancer J Clin. 2018;68(6):394-424.

3. Cai Q, Zhu C, Yuan Y, Feng Q, Feng Y, Hao Y, et al. Development and validation of a prediction rule for estimating gastric cancer risk in the Chinese high-risk population: a nationwide multicentre study. Gut. 2019;68(9):1576-87.

4. Yao K, Uedo N, Kamada T, Hirasawa T, Nagahama T, Yoshinaga S, et al. Guidelines for endoscopic diagnosis of early gastric cancer. Dig Endosc. 2020;32(5):663-98.

5. Dai X, Kaushik AC, Zhang J. The Emerging Role of Major Regulatory RNAs in Cancer Control. Front Oncol. 2019;9:920.

6. Wang T, Liu Y, Zhao M. Mutational analysis of driver genes with tumor suppressive and oncogenic roles in gastric cancer. PeerJ. 2017;5:e3585.

7. Fabbri M, Girnita L, Varani G, Calin GA. Decrypting noncoding RNA interactions, structures, and functional networks. Genome Res. 2019;29(9):1377-88.

8. Chen D, Ping S, Xu Y, Wang M, Jiang X, Xiong L, et al. Non-Coding RNAs in Gastric Cancer: From Malignant Hallmarks to Clinical Applications. Front Cell Dev Biol. 2021;9:732036.

9. Yang G, Lu X, Yuan L. LncRNA: a link between RNA and cancer. Biochim Biophys Acta. 2014;1839(11):1097-109.

10. Chandra Gupta S, Nandan Tripathi Y. Potential of long non-coding RNAs in cancer patients: From biomarkers to therapeutic targets. Int J Cancer. 2017;140(9):1955-67.

11. Yu Y, Chen F, Jin Y, Yang Y, Wang S, Zhang J, et al. Downregulated NORAD in neuroblastoma promotes cell proliferation via chromosomal instability and predicts poor prognosis. Acta Biochim Pol. 2020;67(4):595-603.

12. Li Q, Li C, Chen J, Liu P, Cui Y, Zhou X, et al. High expression of long noncoding RNA NORAD indicates a poor prognosis and promotes clinical progression and metastasis in bladder cancer. Urol Oncol. 2018;36(6):310 e15- e22.

13. Zhang XM, Wang J, Liu ZL, Liu H, Cheng YF, Wang T. LINC00657/miR-26a-5p/CKS2 ceRNA network promotes the growth of esophageal cancer cells via the MDM2/p53/Bcl2/Bax pathway. Biosci Rep. 2020;40(6).

14. Wu X, Lim ZF, Li Z, Gu L, Ma W, Zhou Q, et al. NORAD Expression Is Associated with Adverse Prognosis in Esophageal Squamous Cell Carcinoma. Oncol Res Treat. 2017;40(6):370-4. 
15. Wang L, Du L, Duan W, Yan S, Xie Y, Wang C. Overexpression of long noncoding RNA NORAD in colorectal cancer associates with tumor progression. Onco Targets Ther. 2018;11:6757-66.

16. Luo L, Chen C, He H, Cai M, Ling C. Silencing of Long Non-Coding RNA (LncRNA) Non-Coding RNA Activated by DNA Damage (NORAD) Inhibits Proliferation, Invasion, Migration, and Promotes Apoptosis of Glioma Cells via Downregulating the Expression of AKR1B1. Med Sci Monit. 2020;26:e922659.

17. Miao Z, Guo X, Tian L. The long noncoding RNA NORAD promotes the growth of gastric cancer cells by sponging miR-608. Gene. 2019;687:116-24.

18. Wu Y, Shen QW, Niu YX, Chen XY, Liu HW, Shen XY. LncNORAD interference inhibits tumor growth and lung cancer cell proliferation, invasion and migration by down-regulating CXCR4 to suppress RhoA/ROCK signaling pathway. Eur Rev Med Pharmacol Sci. 2020;24(10):5446-55.

19. Lu J, Getz G, Miska EA, Alvarez-Saavedra E, Lamb J, Peck D, et al. MicroRNA expression profiles classify human cancers. Nature. 2005;435(7043):834-8.

20. Calin GA, Croce CM. MicroRNA signatures in human cancers. Nat Rev Cancer. 2006;6(11):857-66.

21. Liz J, Esteller M. IncRNAs and microRNAs with a role in cancer development. Biochim Biophys Acta. 2016;1859(1):169-76.

22. Ye J, Li J, Zhao P. Roles of ncRNAs as ceRNAs in Gastric Cancer. Genes (Basel). 2021;12(7).

23. Wang $C$, Wu D, He M, Guan L, Bai D, Liang B. LncRNA NORAD accelerates the progression of nonsmall cell lung cancer via targeting miRNA-455/CDK14 axis. Minerva Med. 2021.

24. Yang Y, Zhang G, Li J, Gong R, Wang Y, Qin Y, et al. Long noncoding RNA NORAD acts as a ceRNA mediates gemcitabine resistance in bladder cancer by sponging miR-155-5p to regulate WEE1 expression. Pathol Res Pract. 2021;228:153676.

25. Liu W, Zhou X, Li Y, Jiang H, Chen A. Long Non-Coding RNA NORAD Inhibits Breast Cancer Cell Proliferation and Metastasis by Regulating miR-155-5p/SOCS1 Axis. J Breast Cancer. 2021;24(3):330-43.

26. Sun Y, Wang J, Ma Y, Li J, Sun X, Zhao X, et al. Radiation induces NORAD expression to promote ESCC radiotherapy resistance via EEPD1/ATR/Chk1 signalling and by inhibiting pri-miR-199a1 processing and the exosomal transfer of miR-199a-5p. J Exp Clin Cancer Res. 2021;40(1):306.

27. Yin Y, Zhang B, Wang W, Fei B, Quan C, Zhang J, et al. miR-204-5p inhibits proliferation and invasion and enhances chemotherapeutic sensitivity of colorectal cancer cells by downregulating RAB22A. Clin Cancer Res. 2014;20(23):6187-99.

28. Wang X, Li F, Zhou X. miR-204-5p regulates cell proliferation and metastasis through inhibiting CXCR4 expression in OSCC. Biomed Pharmacother. 2016;82:202-7.

29. Zhang J, Xing L, Xu H, Wang K, She J, Shi F, et al. miR-204-5p Suppress Lymph Node Metastasis via Regulating CXCL12 and CXCR4 in Gastric Cancer. Journal of Cancer. 2020;11(11):3199-206.

30. Zeng J, Wei M, Shi R, Cai C, Liu X, Li T, et al. MiR-204-5p/Six1 feedback loop promotes epithelialmesenchymal transition in breast cancer. Tumour Biol. 2016;37(2):2729-35. 
31. Usui G, Matsusaka K, Mano Y, Urabe M, Funata S, Fukayama M, et al. DNA Methylation and Genetic Aberrations in Gastric Cancer. Digestion. 2021;102(1):25-32.

32. Grady WM, Yu M, Markowitz SD. Epigenetic Alterations in the Gastrointestinal Tract: Current and Emerging Use for Biomarkers of Cancer. Gastroenterology. 2021;160(3):690-709.

33. Cech TR, Steitz JA. The noncoding RNA revolution-trashing old rules to forge new ones. Cell. 2014;157(1):77-94.

34. Sun W, Yang Y, Xu C, Xie Y, Guo J. Roles of long noncoding RNAs in gastric cancer and their clinical applications. J Cancer Res Clin Oncol. 2016;142(11):2231-7.

35. Piao HY, Guo S, Jin H, Wang Y, Zhang J. LINC00184 involved in the regulatory network of ANGPT2 via ceRNA mediated miR-145 inhibition in gastric cancer. Journal of Cancer. 2021;12(8):2336-50.

36. Chen W, Zhang K, Yang Y, Guo Z, Wang X, Teng B, et al. MEF2A-mediated IncRNA HCP5 Inhibits Gastric Cancer Progression via MiR-106b-5p/p21 Axis. Int J Biol Sci. 2021;17(2):623-34.

37. Zhou S, Zhang D, Guo J, Chen Z, Chen Y, Zhang J. Long non-coding RNA NORAD functions as a microRNA-204-5p sponge to repress the progression of Parkinson's disease in vitro by increasing the solute carrier family 5 member 3 expression. IUBMB Life. 2020;72(9):2045-55.

38. Xiong W, Deng Z, Tang Y, Deng Z, Li M. Downregulation of KMT2D suppresses proliferation and induces apoptosis of gastric cancer. Biochem Biophys Res Commun. 2018;504(1):129-36.

39. Noorolyai S, Shajari N, Baghbani E, Sadreddini S, Baradaran B. The relation between PI3K/AKT signalling pathway and cancer. Gene. 2019;698:120-8.

40. Papa A, Pandolfi PP. The PTEN(-)PI3K Axis in Cancer. Biomolecules. 2019;9(4).

41. Lv S, Ji L, Chen B, Liu S, Lei C, Liu X, et al. Histone methyltransferase KMT2D sustains prostate carcinogenesis and metastasis via epigenetically activating LIFR and KLF4. Oncogene. 2018;37(10):1354-68.

\section{Figures}


A

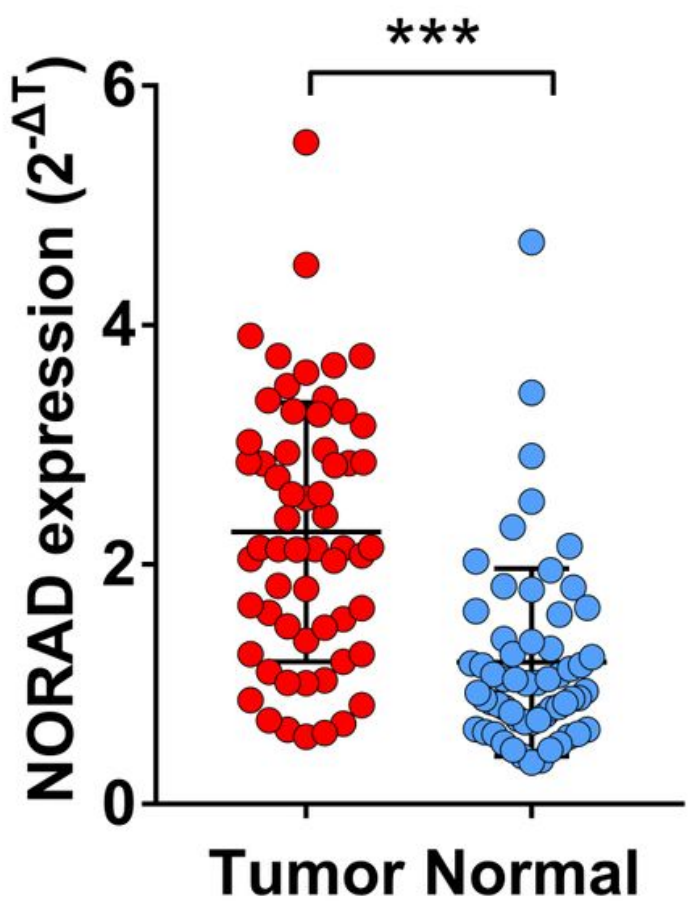

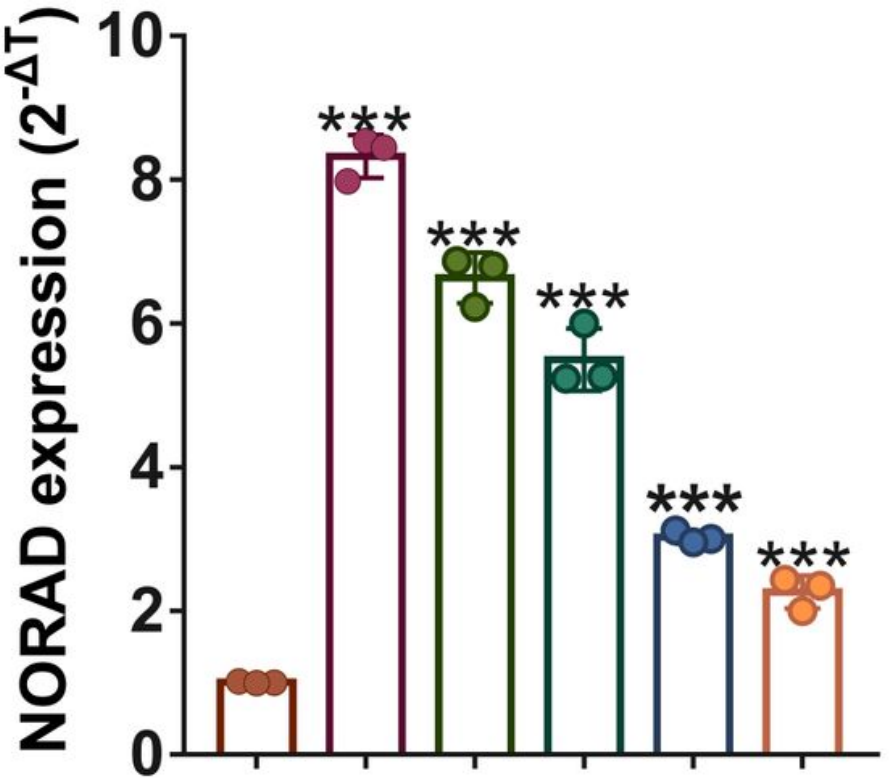

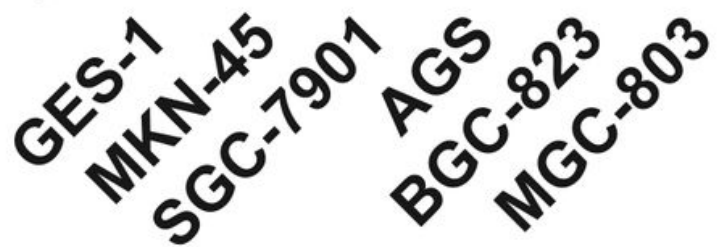

Figure 1

NORAD is up-regulated in GC tissues and cell lines. A Relative expression of NORAD in 60 pairs of GC tumor tissues and adjacent normal tissues was detected by qRT-PCR assay. B The expression levels of NORAD in GC cell lines (MKN-45, SGC-7901, AGS, BGC-823, MGC-803) was relative to the gastric epithelium cell line (GES-1). ${ }^{\star \star \star} P<0.001$. 
A

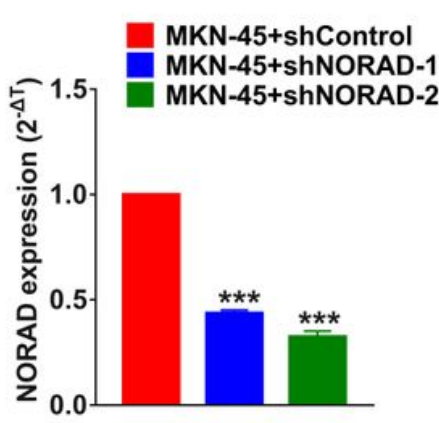

E

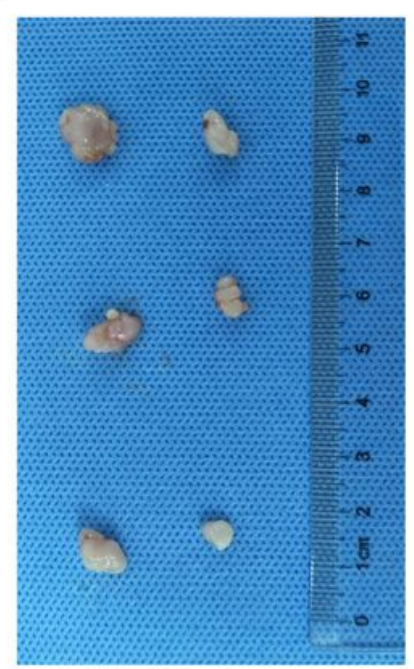

shControl shNORAD-2
B
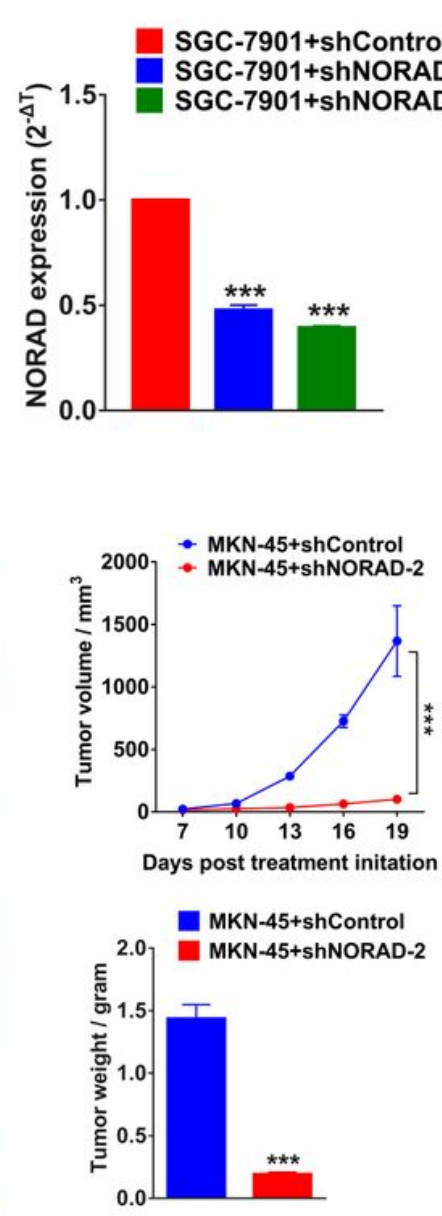

C

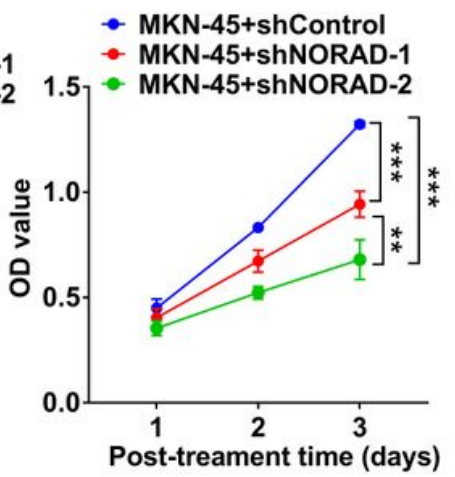

$\mathbf{F}$

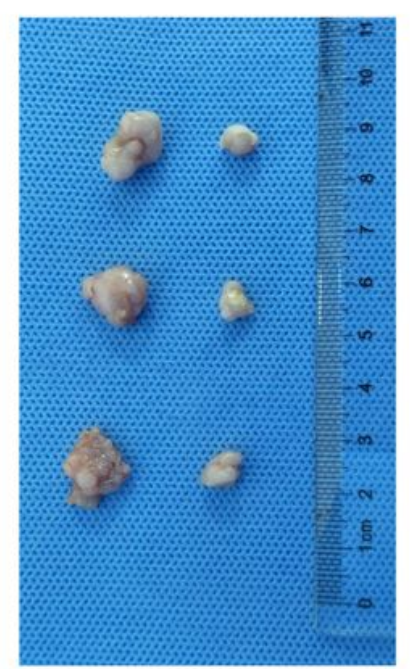

shControl shNORAD-2
D
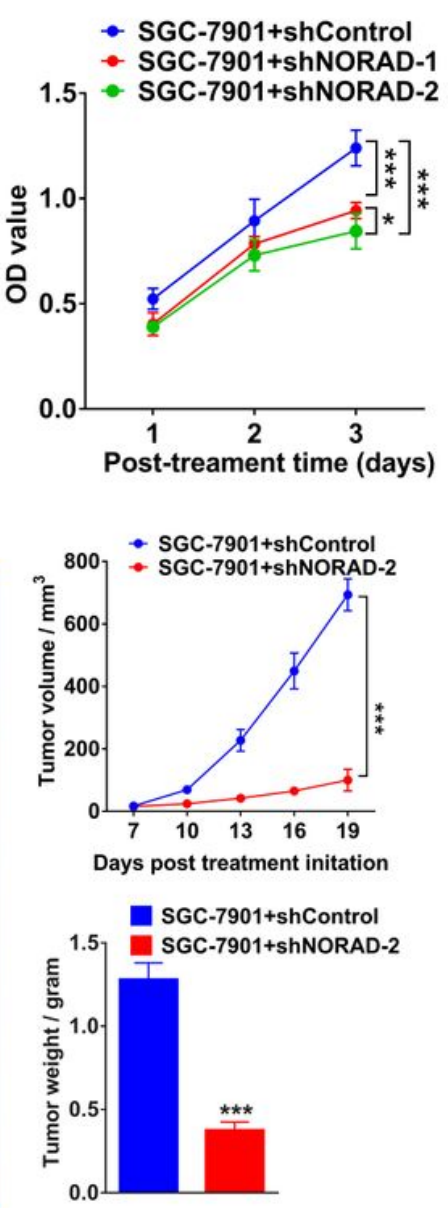

\section{Figure 2}

NORAD silencing hampered GC cell growth. A\&B Relative expression of NORAD was examined in MKN-45 and SGC-7901 cells transfected with shNORAD-1, shNORAD-2 and shControl by qRT-PCR assay. C\&D CCK-8 assay was conducted to detect cell growth in MKN-45 and SGC-7901 cells transfected with shNORAD-1, shNORAD-2 and shControl. E\&F Tumor size (left panel), tumor weight (middle panel), and tumor volumes (right panel) of xenografts harvested at 18 days after subcutaneous injection of MKN-45

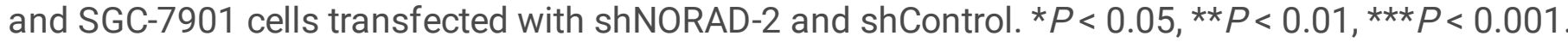




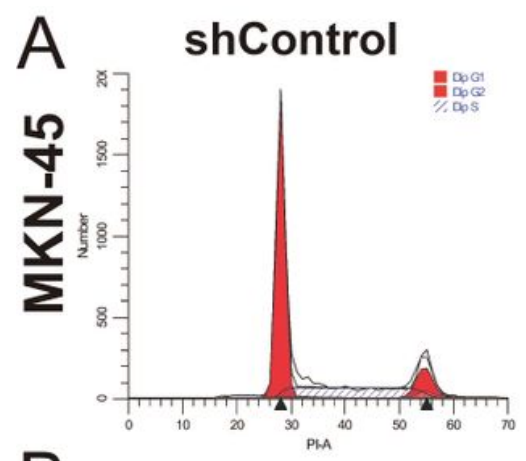

shNOARD-1
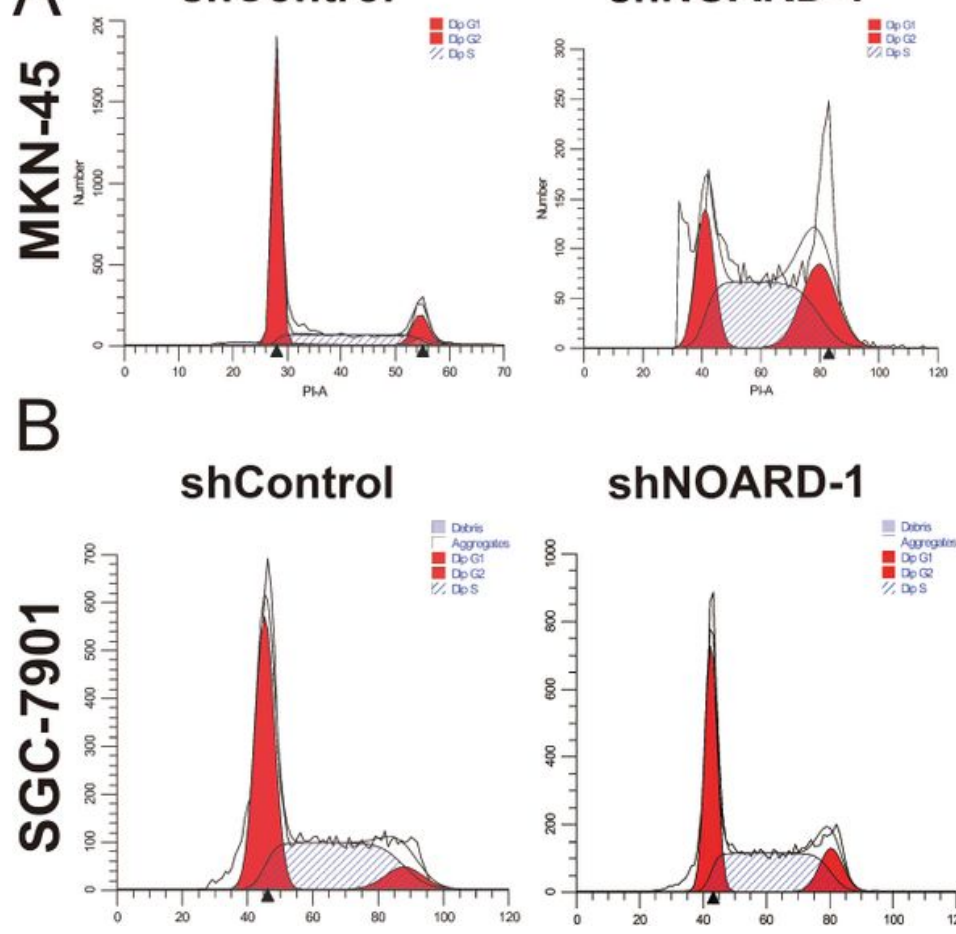

shNOARD-1

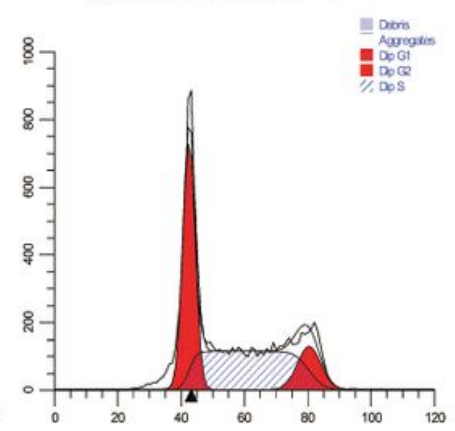

ShNOARD-2

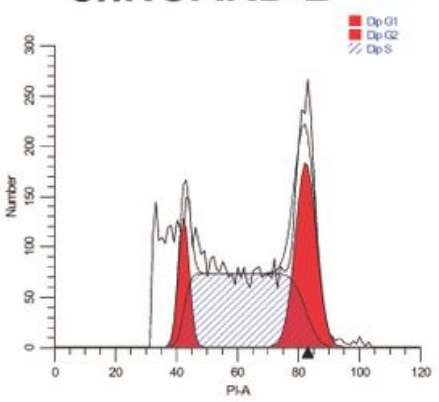

shNOARD-2

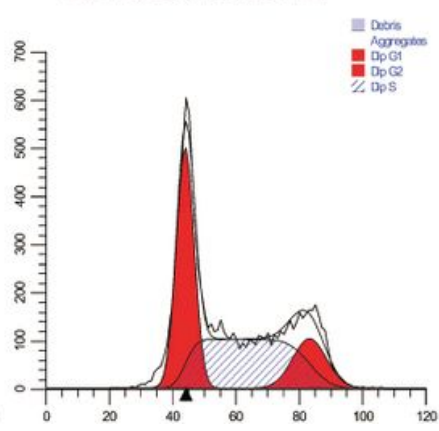

MNK-45+shContro

MNK-45+shNORAD-1
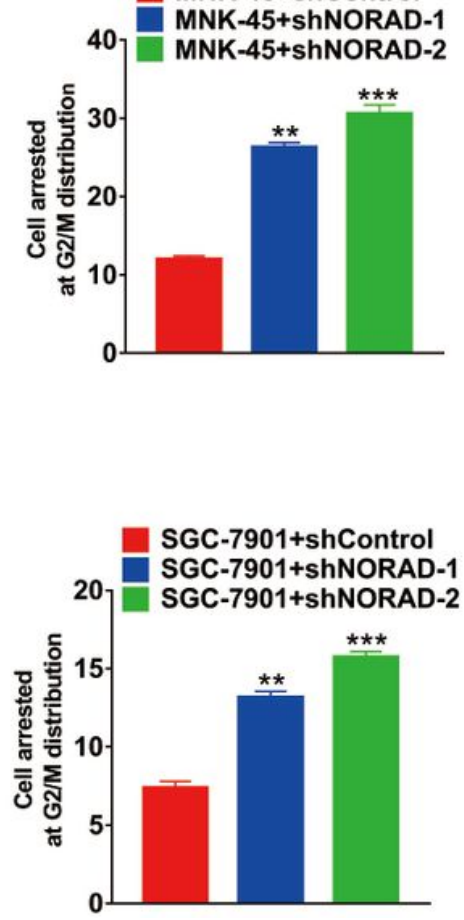

Figure 3

NORAD silencing led to G2/M cell cycle arrest in GC. Flow cytometric analysis of mitotic cell cycle of MKN-45 and SGC-7901 cells transfected with shNORAD-1, shNORAD-2 and shControl (left panel). Quantification analysis of GC cells arrested in G2/M phase was shown (right panel). 
A

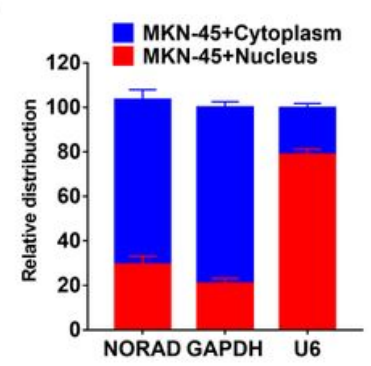

D

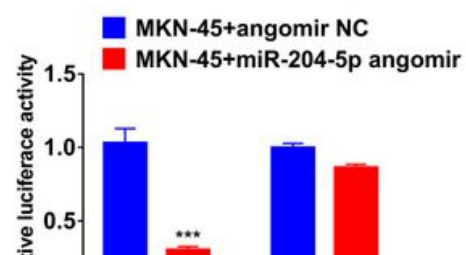

B

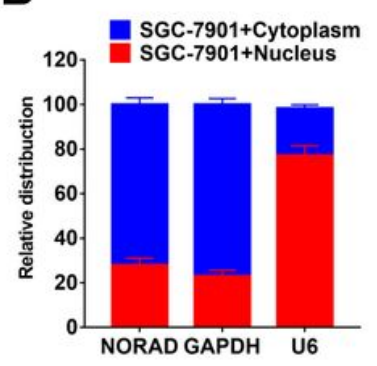

E

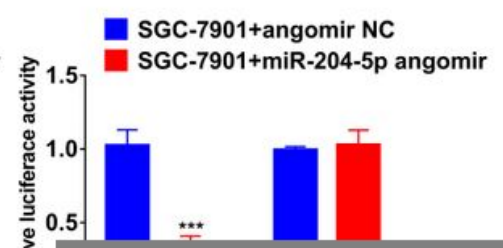

C

miR-204-5p 5' - UCCGUAUCCUACUGUUUCCCUU - 3'

| | || || ||||||

NORAD 3'- UGGUAGAGG -UGGGAAAGGGAA - 5
F $\quad$ G

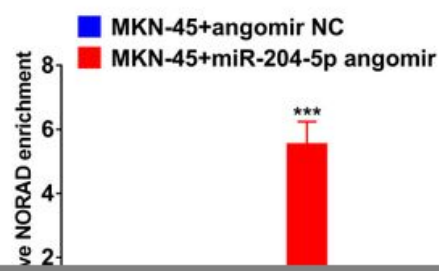

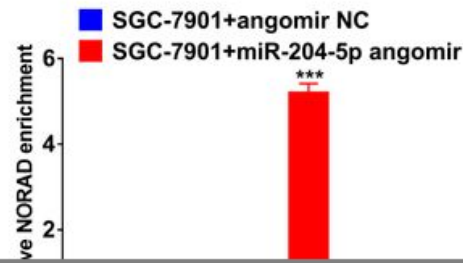

Figure 4

NORAD directly targeted miR-204-5p in GC. A\&B, Nuclear and cytoplasmic expression of NORAD was examined in MKN-45 and SGC-7901 cells by qRT-PCR. C, Putative complementary sites between NORAD and miR-204-5p. D\&E Luciferase activity of MKN-45 and SGC-7901 cells co-transfected with miR-204-5p angomir or angomir NC with NORAD WT or NORAD MUT transcripts. F\&G Relative enrichment of NORAD was measured in MKN-45 and SGC-7901 cells transfected with miR-204-5p angomir or angomir NC by anti-Ago2 RNA immunoprecipitation assay. H\&I Relative expression of miR-204-5p was examined in MKN45 and SGC-7901 cells transfected with shNORAD-1, shNORAD-2 and shControl. J Relative expression of miR-204-5p in 60 pairs of GC tumor tissues and adjacent normal tissues was detected by qRT-PCR assay. K Statistical correlation between NORAD and miR-204-5p in GC tissue was analyzed by Pearson's correlation coefficient. ${ }^{\star *} P<0.01, \star \star \star * P<0.001$. 


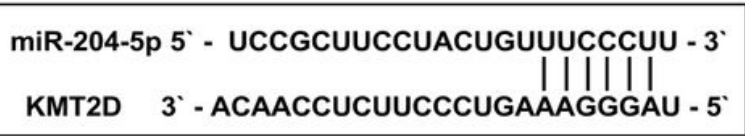

miR-204-5p 5' - UCCGCUUCCUACUGUUUCCCUU - 3' KMT2D $3{ }^{\prime}$ - ACAACCUCUUCCCUGAAAGGGAU - 5

D

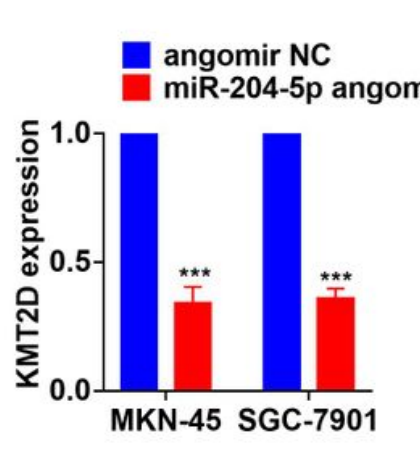

G

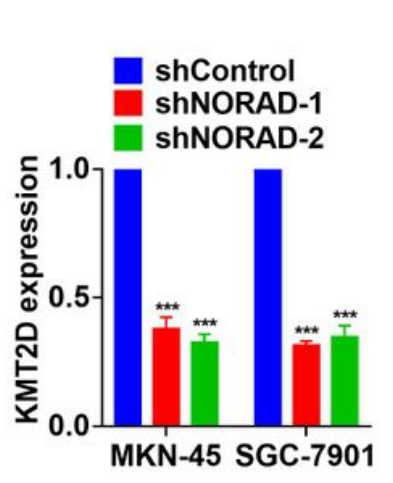

E

H
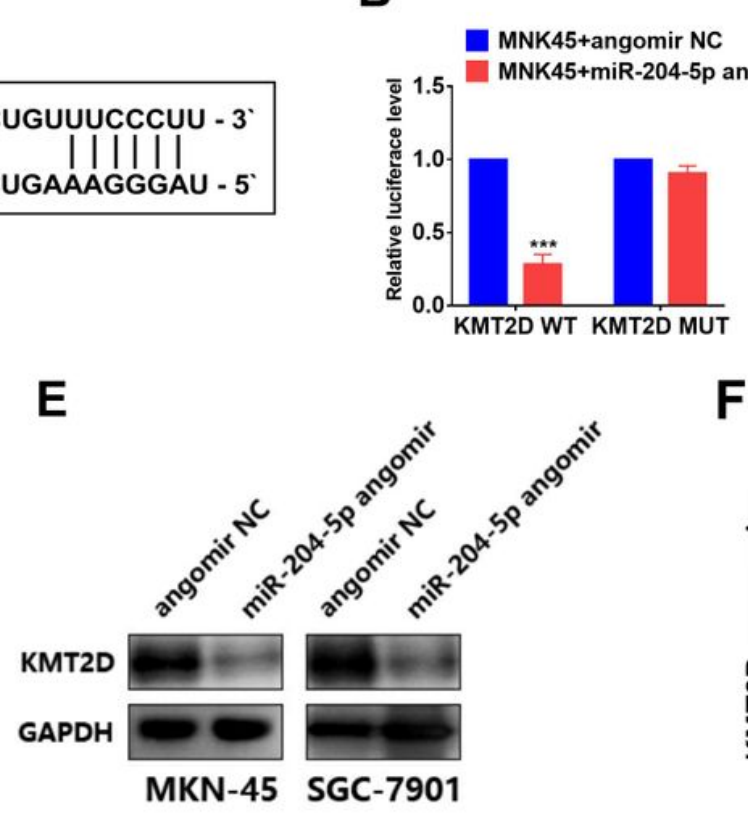

$\mathbf{F}$

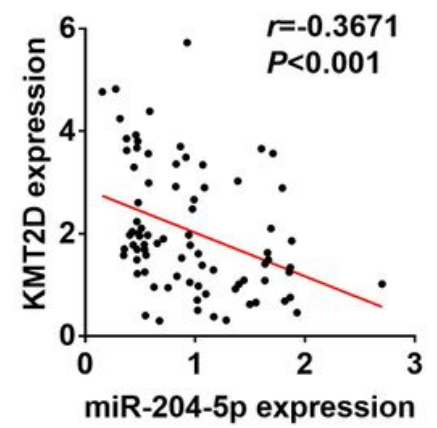

I
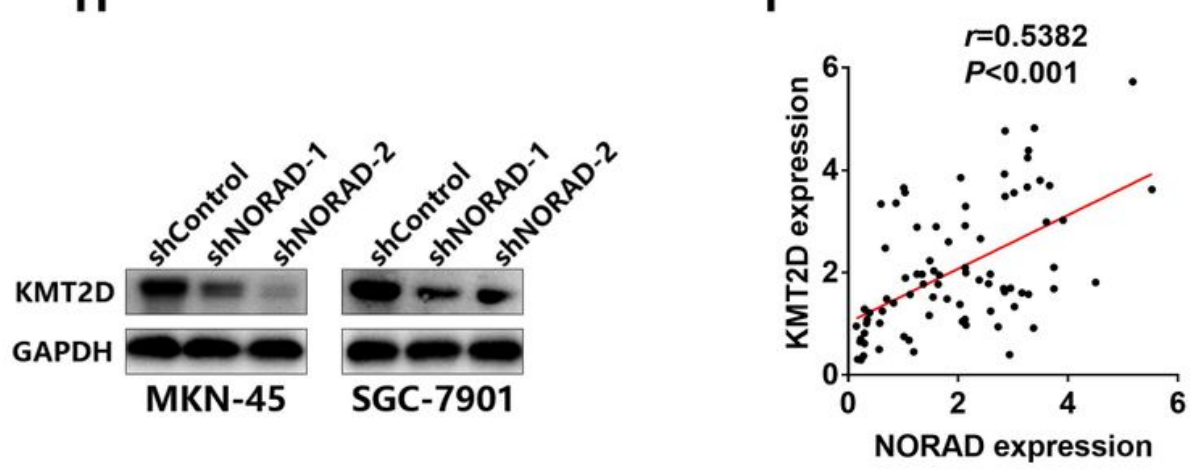

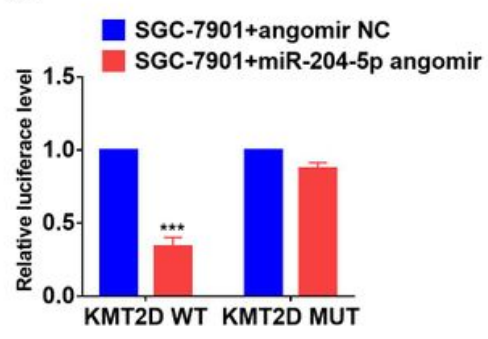

SGC-7901+angomir NC

Figure 5

KMT2D was a direct downstream target of miR-204-5p in GC. A Putative complementary site between KMT2D and miR-204-5p. B\&C Luciferase activity of MKN-45 and SGC-7901 cells co-transfected with miR204-5p angomir or angomir NC with KMT2D WT or KMT2D MUT transcripts. D Relative expression of KMT2D was examined in MKN-45 and SGC-7901 cells transfected with miR-204-5p angomir or angomir NC by RT-qPCR. E Expression of KMT2D was examined in MKN-45 and SGC-7901 cells transfected with miR-204-5p angomir or angomir NC by Western blot. F Statistical correlation between KMT2D and miR204-5p in GC tissue was analyzed by Pearson's correlation coefficient. G Relative expression of KMT2D was examined in MKN-45 and SGC-7901 cells transfected with shNORAD-1, shNORAD-2 and shControl by RT-qPCR assay. H Expression of KMT2D was examined in MKN-45 and SGC-7901 cells transfected with shNORAD-1, shNORAD-2 and shControl by Western blot. I Statistical correlation between KMT2D and NORAD in GC tissue was analyzed by Pearson's correlation coefficient. ${ }^{\star \star \star} P<0.001$. 
A

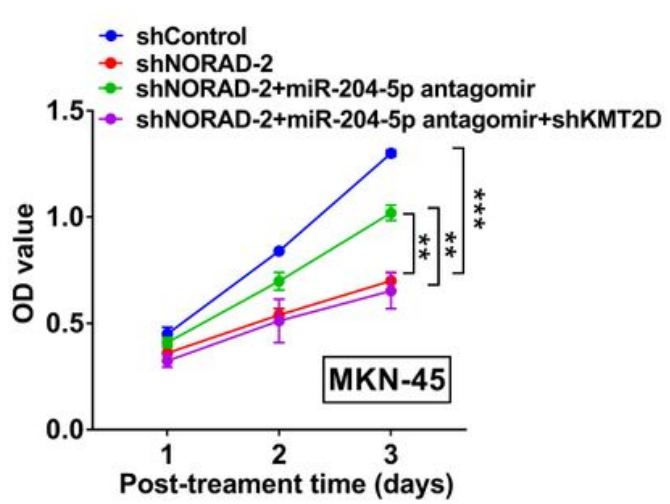

B

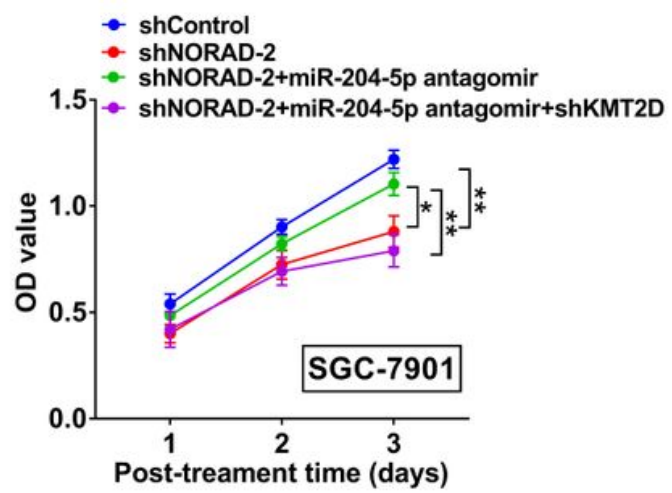

ShNOARD-2+

shNOARD-2+ miR-204-5p antangomir

C shControl ShNOARD-2 miR-204-5p antangomir +shKMT2D

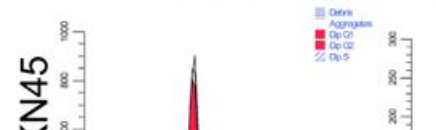<smiles>C=CC=CC=C</smiles>

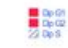

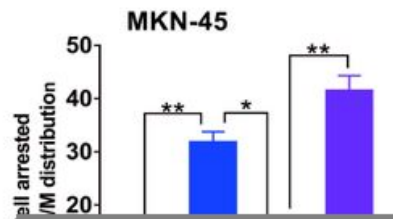

\section{Figure 6}

NORAD regulated GC growth by targeting miR-204-5p/KMT2D axis. A CCK-8 assay was conducted to detect cell growth in MKN-45 and SGC-7901 cells after co-transfection. B Flow cytometric analysis of mitotic cell cycle of MKN-45 and SGC-7901 cells after co-transfection. Quantification analysis of GC cells arrested in G2/M phase was shown (right panel). ${ }^{\star} P<0.05$, ${ }^{\star} P \mathrm{P}<0.01$, ${ }^{\star \star \star} P<0.001$. 
MKN-45

\section{PTEN}

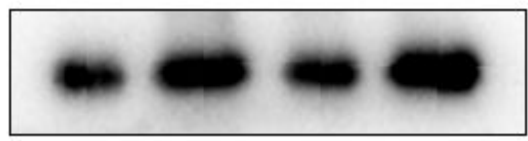

PI3K

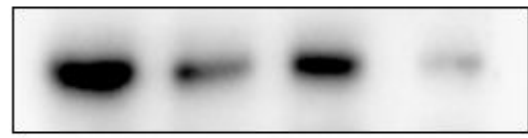

AKT

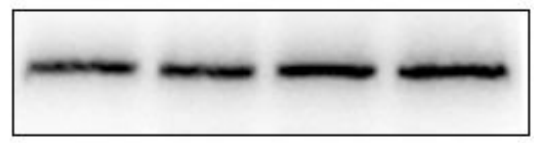

p-AKT

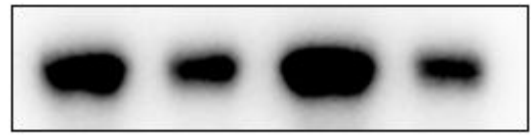

GAPDH

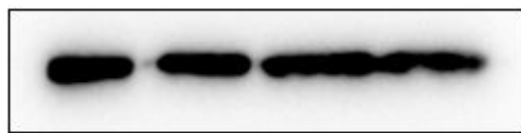

shNORAD-2 - $+-\quad+$

miR-204-5p antagomir shKMT2D
SGC-7901
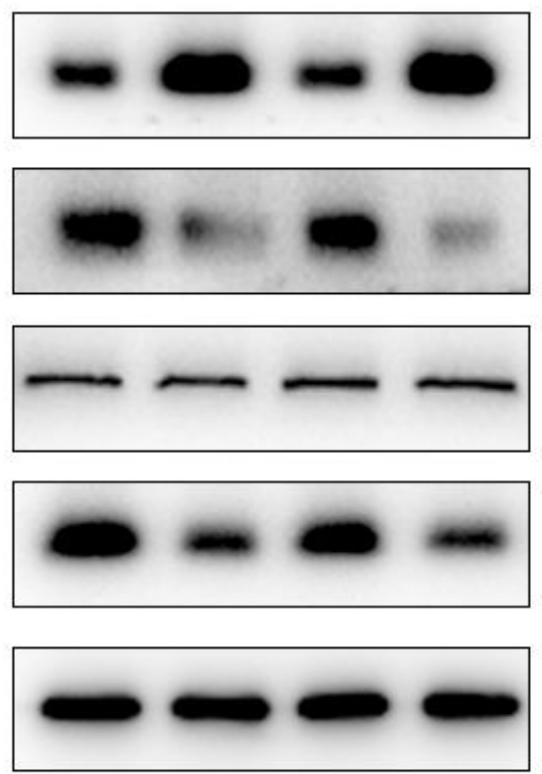

$-\quad+\quad+$

$-\quad+\quad+$

$-\quad+\quad+$

Figure 7

NORAD regulated PTEN/PI3K/AKT pathway by targeting miR-204-5p/KMT2D axis in GC. Expression levels of PTEN, PI3K, AKT and PAKT protein was detected in MKN-45 and SGC-7901 cells after cotransfection by Western blot. 


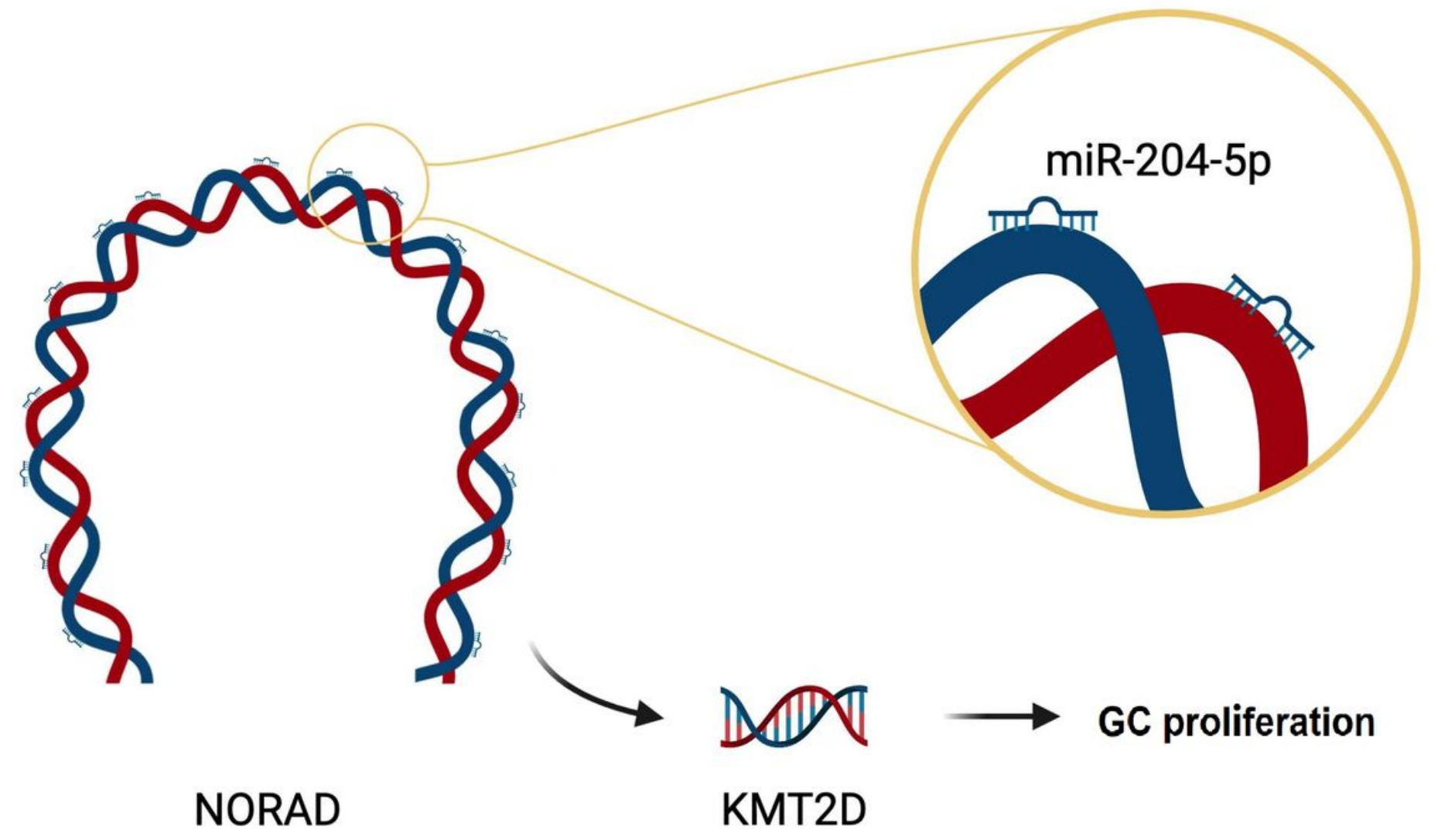

Figure 8

Schematic illustration of the regulation of GC progression by the NORAD /miR-204-5p/KMT2D axis.

\section{Supplementary Files}

This is a list of supplementary files associated with this preprint. Click to download.

- FigureS1.jpg 